\title{
Comparando dos métodos de estimación del tamaño de las redes personales ${ }^{1}$
}

\author{
Christopher McCarty², Peter D. Killworth, H. Russell Bernard, Eugene C. Johnsen y
}

Gene A. Shelley

\section{Resumen}

En este artículo comparamos dos métodos para la estimación del tamaño de las redes personales utilizando una muestra representativa de Estados Unidos a nivel nacional. Ambos métodos se basan en la habilidad de las personas encuestadas para estimar el número de personas que conocen en subpoblaciones específicas de EE.UU. (ej.: diabéticos, nativo-americanos) y gente en categorías específicas de relación (ej.: familia inmediata, compañeros de trabajo). Los resultados muestran una remarcable similitud entre el tamaño medio de la red obtenido por ambos métodos (aproximadamente 291). Se obtuvieron resultados similares con una muestra nacional distinta. La tentativa de corroboración de nuestras estimaciones mediante una reproducción exacta de la encuesta entre un segmento de población propenso a tener redes más amplias (el clero), dio como resultado un tamaño medio de la red superior.

Una investigación extensiva sobre la existencia de efectos de respuesta mostró algunas preferencias por usar ciertos números a la hora de realizar estimaciones, pero nada que afectase de forma significativa a la estimación de tamaño de la red más allá del 6 por ciento. Nuestra conclusión es que ambos métodos utilizados para la estimación del tamaño de las redes personales proporcionan resultados válidos y fiables del tamaño de la red real, pero quedan algunas cuestiones pendientes sobre la exactitud.

Palabras clave: Tamaño de la red, poblaciones difíciles de estimar, encuesta telefónica.

\begin{abstract}
In this paper we compare two methods for estimating the size of personal networks using a nationally representative sample of the United States. Both methods rely on the ability of respondents to estimate the number of people they know in specific subpopulations of the U.S. (e.g., diabetics, Native Americans) and people in particular relation categories (e.g., immediate family, coworkers). The results demonstrate a remarkable similarity between the average network size generated by both methods (approximately 291). Similar results were obtained with a separate national sample. An attempt to corroborate our estimates by replication
\end{abstract}

\footnotetext{
1 Traducción de la publicación original: McCarty, Christopher, Killworth, Peter D., Bernard, Russel H., Johnsen, Eugene C. \& Shelley, Gene A. (2001). Comparing Two Methods for Estimating Network Size, Human Organization, 60, 28-39.

2 Enviar correspondencia a: (chrism@bebr.ufl.edu). Chris McCarty es director de encuestas de la Oficina de economía e investigación empresarial (Bureau of Economic and Business Research) de la Universidad de Florida, Gainsville; Peter Killworth es un científico de reconocido prestigio en el Centro Oceanográfico de Southhampton (Southampton Oceanography Centre), Southhampton, UK.; Russ Bernard es catedrático de antropología en la Universidad de Florida, Gainesville; Eugene Johnsen es catedrático emérito de matemáticas en la Universidad de California, Santa Bárbara; y Gene Shelley pertenece a la Georgia State University, Atlanta. Nuestro agradecimiento a John Domini, Amber Yoder y David Kennedy por su ayuda con la recopilación de datos y durante la redacción de este estudio. La Oficina de economía e investigación empresarial de Florida nos suministró los servicios de encuesta telefónica así como un lugar para que pudiéramos trabajar durante la crítica del análisis y la redacción. El trabajo ha recibido la financiación de la beca NSF SBR-9710353.
} 
among a population we suspect has large networks (clergy), yielded a larger average network size.

Extensive investigation into the existence of response effects showed some preference for using certain numbers when making estimates, but nothing that would significantly affect the estimate of network size beyond about 6 percent. We conclude that both methods for estimating personal network size yield valid and reliable proxies for actual network size, but questions about accuracy remain.

Key words: network size, hard-to-count populations, telephone survey.

Desde 1986, hemos estado trabajando en un método para estimar la distribución del tamaño de las redes personales entre la población de EE UU y el tamaño de las subpoblaciones difíciles de estimar, como pueden ser los seropositivos, los sin techo o las víctimas de violación. Un componente principal de nuestro método incluye la estimación del tamaño medio de las redes personales en una muestra extensa de población usando lo que llamamos un "método de escalamiento proporcional". Sólo se han realizado un puñado de estudios de estimación del tamaño de las redes personales, que incluyen los estudios Reverse Small-World ("El Pequeño Mundo al revés", Bernard, Killworth, and McCarty 1982; Killworth y Bernard 1978), los estudios de la agenda de teléfonos (Freeman y Thompson 1989), nuestras tentativas iniciales (Bernard et al. 1989; Killworth et al. 1990; Bernard et al. 1991; Johnsen et al. 1995) y los actuales estudios de método de escalamiento proporcional (Killworth et al. 1998a, 1998b). Estos métodos ofrecen estimaciones muy variadas de tamaño de la red, en parte debido a la definición de quién debería incluirse en la red de las personas encuestadas y a las características de los propios métodos.

Este artículo examina de forma crítica nuestra metodología hasta hoy e introduce una extensión a un segundo método paralelo de "suma". Comenzaremos describiendo brevemente el método de escalamiento proporcional y luego definiremos el nuevo método de suma. Se compararn los resultados de ambos métodos para descubrir que son similares. Y a continuación discutiremos los hallazgos realizados a partir del trabajo con grupos focales que sugieren varios posibles efectos de confusión en nuestros métodos. Éstos incluyen: la preferencia de los encuestados por ciertos números; la posibilidad de que la coherencia entre métodos y entre encuestas sea una ilusión producto de nuestras aproximaciones numéricas; los datos omitidos; la posibilidad que las personas encuestadas elegidas por su red de gran tamaño modifiquen el resultado y la posibilidad que los encuestados sean menos capaces de estimar de forma exacta para subpoblaciones extremamente pequeñas y extremamente grandes. 


\section{El método de escalamiento proporcional}

Nuestro método se basa en la presunción de que el número de gente que una persona conoce dentro de una subpoblación determinada es una función de, entre otras cosas, el número de gente conocida en general. El método parte de una presunción que ha sido sometida a una serie de pruebas en Killworth et al. (1998a, 1998b). Asume que, entre otras cosas iguales, la probabilidad de que cualquier miembro de la red de un encuestado se encuentre en una subpoblación es una fracción de la población mayoritaria ocupada por esta subpoblación (en otras palabras, si un 1/100 de la población de EEUU posee alguna característica, como promedio uno debe asumir que un 1/100 de cualquier red posee también esta característica).

De forma más formal, pongamos que $\mathrm{m}$ es el número de gente teóricamente conocida en una subpoblación E (por ejemplo, víctimas de violación) de una población más grande $T$ (como la población de EEUU), c es el tamaño de la red personal del encuestado, $\mathrm{t}$ el tamaño de $\mathrm{T}$ y e el tamaño de la subpoblación. La probabilidad de que cualquier miembro de c esté en $\mathrm{E}$ es e/t, por lo que el número esperado conocido vendría dado por:

$$
\frac{m}{c}=\frac{e}{t}
$$

No esperamos que esta proporcionalidad tan simple se aplique a un individuo específico: el número de gente que los encuestados dicen conocer en la subpoblación tiene una distribución binómica con la probabilidad $p=e / t$ y significa cp. Se pregunta al encuestado sobre muchas subpoblaciones y de las respuestas obtenidas, se computa la estimación máxima posible de la c del encuestado (o lo que es lo mismo: una que se ajuste mejor al patrón de respuestas obtenido). Una vez estimado el tamaño de la red de cada encuestado (y la distribución de ésta es de una gran interés teórico), una estimación de probabilidad máxima similar se computa para la parte fraccionaria de las subpoblaciones desconocidas. Cada encuestado tiene un valor c y explica cuántas personas conoce en la subpoblación desconocida. La estimación de probabilidad máxima se construye a partir del patrón de respuestas de todos los encuestados.

El método de escalamiento proporcional se basa en otras tres presunciones: 
1. Todo el mundo en T tiene la misma posibilidad de conocer a alguien en E; es decir, todos los habitantes de EEUU tienen la misma posibilidad de conocer a una persona diabética, por ejemplo. Esta posibilidad aumenta de forma proporcional con el tamaño de E.

2. Todo el mundo tiene un conocimiento exacto de toda la gente que conoce. Es decir, si alguien de su lista de miembros de la red social es diabético, lo saben. También saben si un miembro de su red tiene un gemelo, ha sufrido la fiebre tifoidea, se gana la vida como constructor, tiene una tarjeta de crédito American Express, etc.

3. Los encuestados pueden decirnos de forma exacta y en muy poco tiempo (menos de 30 segundos) cuánta gente conocen que sean, por ejemplo, nativo-americanos, diabéticos o golfistas ("conocer" a alguien se define de la siguiente manera: "uno conoce a la persona y ésta le conoce a uno de vista o por el nombre; puede contactar con ella en persona, por teléfono o por correo y se ha tenido contacto con esa persona los últimos dos años").

Llamamos a una violación de la primera presunción "efecto de barrera". Es decir, existen características espaciales y sociodemográficas de los encuestados y de la gente que conocen que crean barreras para algunos encuestados a la hora de conocer a cierto tipo de personas ( $\mathrm{y}$ al contrario, los encuestados que forman parte de una subpoblación tienen más posibilidades de conocer a otros miembros de esta subpoblación). Sin embargo, en una muestra de encuestados representativa a nivel nacional y para los tamaños de subpoblaciones utilizados aquí, es fácil mostrar que este aspecto tiene un efecto insignificante sobre los cálculos.

Llamamos a una violación de la segunda presunción "efecto de transmisión". Es decir, sabemos que el hecho de pertenecer a una subpoblación no se transmite con las mismas probabilidades a todos los miembros de la red (a la gente que conocen). Esto se debe tanto a: 1) el estigma o vergüenza asociado con la pertenencia a éste; 2) porque no es un tema común de conversación; o 3) es personal y privado y normalmente ni siquiera se discute con amigos (normalmente no se divulga la riqueza de uno aunque puede ser objeto de especulación entre amigos y conocidos). Los efectos de barrera y transmisión se investigan en un estudio a parte. Llamamos a una violación de la tercera presunción "efecto de estimación" y la detallamos más adelante. 
Otra razón por la que puede que los encuestados no sean capaces de calcular de forma exacta el número de gente que conocen en una subpoblación particular es que no estén seguros de los límites de esta subpoblación; es decir, el término es ambiguo. Por ejemplo, un encuestado puede conocer a alguien que comenzó a realizar diseños de páginas web en su casa el año pasado pero no está seguro de que esa persona cuenta como "persona que ha abierto un negocio en los últimos 12 meses". De hecho, la definición usada por aquéllos que recopilaron el Resumen Estadístico de EEUU (U.S. Statistical Abstract) es muy específica y reflejaría datos disponibles de fuentes tales como registros de aquéllos que se han incorporado. Como los encuestados no suelen tener conocimiento de estas definiciones, o es posible que no las interpreten sistemáticamente, esto puede afectar a la exactitud de nuestras estimaciones.

El alcance de este error varía en gran medida dependiendo de la subpoblación en particular. El abanico de definiciones de "sin techo" (véase, por ejemplo, la Oficina del Censo de EEUU) demuestra lo difícil que sería para los encuestados definir a esta subpoblación de forma no ambigua. Lo mismo se puede aplicar a otras subpoblaciones, como los nativo-americanos, o en el caso de México, a su población indígena. Por otro lado, algunas subpoblaciones son fáciles de definir, como los diabéticos o las mujeres que han dado a luz en el último año.

El problema de la ambigüedad de los límites de los grupos de subpoblación a los ojos de los encuestados es, en gran medida, inevitable. Nos vemos limitados a subpoblaciones para las que tenemos cifras. Y a menudo, las definiciones usadas por las entidades de gobierno que las recogen son demasiado específicas para que un encuestado las entienda. Aún más, si confiamos en subpoblaciones definidas de forma no ambigua, es posible encontrarnos con que son de un tipo específico, como una enfermedad o eventos bien definidos. Esto puede introducir una fuente de error ya que algunas personas podrían ser más propensas a conocer gente en estas subpoblaciones específicas. En otras palabras, el beneficio de usar subpoblaciones de distintos tipos se puede ver perjudicado.

La ambigüedad de las preguntas de la encuesta no es algo exclusivo de nuestra investigación. La mayoría de los textos en estudios de encuestas y la construcción de los cuestionarios enfatizan la importancia de evitar términos y expresiones ambiguas. Los estudios han mostrado que en algunos casos el efecto puede ser significativo (Fowler 1992:218). Deberíamos apuntar que el efecto ambigüedad es relevante tanto para los casos de escalamiento proporcional como los métodos de suma. En algunos casos, los encuestados puede que estén inseguros (o que no 
estén de acuerdo) sobre si alguien que conocen se ajusta a una tipo de relación particular (como alguien que conocen a través de una afición o una organización) ${ }^{3}$.

En la primera encuesta nacional telefónica de 1.554 encuestados de todos los EEUU en la que aplicamos nuestro método de escalamiento proporcional, pedimos a los encuestados que estimaran el número de gente que conocían en 29 subpoblaciones de tamaño conocido, tales como gente con un nombre de pila particular, víctimas de accidentes con vehículos de motor y diabéticos. La Tabla 1 muestra la lista de las 29 subpoblaciones.

Esta encuesta generó una media de tamaño de la red de 286 (Killworth et al. 1998b). Tenemos la confianza de que el método de escalamiento proporcional produce una estimación útil y razonable para tamaño de la red, pero existen problemas. Por un lado, existe una tendencia aparente a que los encuestados exageren sobre el número de gente que conocen para subpoblaciones pequeñas y en cambio, subestimen la cifra para las subpoblaciones grandes. Esto se debe, en parte, a la dependencia de las tres presunciones detalladas anteriormente.

\footnotetext{
${ }^{3}$ Para el método de suma, no es importante si los encuestados están de acuerdo o no quién está o no dentro de una categoría de relación mientras lleguen a la suma total. Para el enfoque de escalamiento proporcional, es esencial que los encuestados utilicen la misma definición de subpoblación.
} 


\begin{tabular}{|c|c|c|c|c|c|}
\hline \multicolumn{2}{|c|}{$\begin{array}{l}29 \text { Poblaciones por el método } \\
\text { de escalamiento proporcional }\end{array}$} & \multicolumn{2}{|l|}{$\begin{array}{l}16 \text { Tipos de relación por el } \\
\text { método de suma }\end{array}$} & \multicolumn{2}{|c|}{$\begin{array}{c}3 \text { Poblaciones para ser } \\
\text { estimadas }\end{array}$} \\
\hline Michael & 4.8 & Familia inmediata & 3.5 & Tienen VIH & 0.7 \\
\hline Christina & 1.3 & Otros familiares de nacimiento & 4.0 & Mujeres violadas en & \\
\hline Christopher & 1.8 & Familia de la esposa o pareja & 12.3 & los últimos 12 meses & 0.2 \\
\hline J acqueline & 0.7 & Compañeros de trabajo & 35.6 & Sin techo & 0.7 \\
\hline James & 3.4 & Gente con la que trabaja, & & & \\
\hline Jennifer & 2.3 & aunque no directamente & 62.1 & & \\
\hline Anthony & 1.7 & Mejores amigos/confidentes & 4.3 & & \\
\hline Kimberly & 1.4 & Gente que conoce por & & & \\
\hline Robert & 4.1 & aficiones/ocio & 12.3 & & \\
\hline Stephanie & 1.3 & Gente de organización religiosa & 43.4 & & \\
\hline David & 3.5 & Gente de otra organización & 17.1 & & \\
\hline Nicole & 1.1 & Relaciones escolares & & & \\
\hline Nativos americanos & 3.5 & Vecinos & 12.8 & & \\
\hline Han dado a luz en los últimos & & Sólo amigos & 22.6 & & \\
\hline 12 meses & 3.6 & Gente conocida por terceros & 22.6 & & \\
\hline Viudas/os de menos & & Relaciones de la infancia & 6.8 & & \\
\hline de 65 años & 3.2 & Gente que suministra un servicio & 7.7 & & \\
\hline En diálisis & 0.6 & Otros & 3.9 & & \\
\hline Empleado en correos & 2.2 & & & & \\
\hline Piloto comercial & 0.7 & & & & \\
\hline Miembro de Jaycees & 1.1 & & & & \\
\hline Diabético & 3.3 & & & & \\
\hline Ha abierto un negocio en los & & & & & \\
\hline últimos 12 meses & 1.1 & & & & \\
\hline Tiene un hermano o hermana & & & & & \\
\hline gemelo & 2.0 & & & & \\
\hline Vende armas con licencia & 0.5 & & & & \\
\hline Tiene SIDA & 0.4 & & & & \\
\hline Hombres en prisión Estatal & & & & & \\
\hline o federal & 1.0 & & & & \\
\hline Víctima de homicidio en los & & & & & \\
\hline últimos 12 meses & 0.2 & & & & \\
\hline Se ha suicidado en en los & & & & & \\
\hline últimos 12 meses & 0.2 & & & & \\
\hline $\begin{array}{l}\text { Ha muerto en accidente de aut } \\
\text { móvil en los últimos } 12 \text { meses }\end{array}$ & 0.5 & & & & \\
\hline
\end{tabular}

Tabla 1. Promedio de gente conocida por subpoblaciones y tipos de relación (encuestas combinadas sobre un total de 1.370 encuestados)

\section{El Método de suma}

Las presunciones de nuestro modelo sugieren un programa de investigaciones, cuyo objeto es en el mejor de los casos estimar de forma exacta y en el peor, minimizar los efectos de barrera, transmisión y estimación y tratar de corregirlos. Esto mejoraría la exactitud de nuestra estimación de tamaño de la red y nuestras estimaciones de tamaños de subpoblación. Nuestros esfuerzos corrientes a lo largo de estas líneas se detallaran en otro lado. Para minimizar los efectos de transmisión y barrera, hemos puesto a prueba un método completamente distinto de estimación de tamaño de la red. En lugar de pedir a la gente que cuenten sus alteri de red que pertenecen a varias subpoblaciones, hemos pedido a la gente que cuente el número de esos alteri que mantienen varias relaciones con los encuestados (familia, 
trabajo, etc.). La lista de los 16 tipos de relación se muestra también en la Tabla 1 ( hay que apuntar que la categoría "Otros" engloba los miembros de la red que no han sido calificados de otra forma).

Utilizar tipos de relación para estimar el tamaño de la red en lugar de subpoblaciones computables ofrece varias ventajas potenciales:

1. A los encuestados les puede resultar más fácil hacer estimaciones más pequeñas que tener que distribuir a toda la gente que conocen por subpoblaciones determinadas.

2. Este método es más rápido de implementar porque debe recoger al momento 20-30 subpoblaciones de tamaño conocido para estimar el tamaño de la red, c.

3. El método de escalamiento proporcional depende de un cálculo exacto de algunas subpoblaciones. Éstos son difíciles de obtener, en particular en los países en vías de desarrollo.

4. Elimina de forma virtual los efectos de transmisión y barrera de la estimación de c. Los encuestados casi siempre saben quién constituye y quién no un tipo específico de relación (p.ej: relación familiar, relación laboral, etc.) mientras que no tienen por qué saber si alguien es diabético o nativo americano (véase presunción 2, más arriba). Además, no esperamos barreras espaciales o sociodemográficas para miembros conocidos de la red de un tipo de relación particular, y esto elimina el efecto barrera.

5. El método de suma es independiente del de escalamiento proporcional para estimar c, que utiliza las estimaciones de los encuestados de subpoblaciones de tamaños conocidos para subpoblaciones de tamaños desconocidos.

Brewer (1993, 1995a, 1995b) y Brewer y Yang (1994) también presentan pruebas de que para los encuestados sería más fácil informar sobre el campo "tipos de relación".

Utilizar los tipos de relación para estimar el tamaño de la red presenta algunas desventajas potenciales: 
1. No hay forma de comprobar la validez de las estimaciones de los encuestados sobre el número de gente que conocen en varias categorías de relación ya que los tamaños de estas categorías son desconocidos. El método de escalamiento proporcional se basa en subpoblaciones computables, lo que proporciona una forma de comprobar la integridad estadística de las estimaciones de c (véase Sudman, Bradburn y Schwarz 1996 por su estudio sobre computación contra estimación en las encuestas).

2. Contar más de una vez a un miembro de una red (por ejemplo como relación laboral y como alguien con quien el encuestado socializa) puede ser común. Este tipo de error sistemático podría resultar en una inflación de c y en una deflación en la estimación de las poblaciones desconocidas.

\section{Diseño de encuesta}

Probamos y comparamos los métodos de escalamiento proporcional y de suma para estimar el tamaño de las redes personales en cuatro encuestas telefónicas nacionales en EEUU (Encuesta 1, $\mathrm{n}=796$, enero 1998, porcentaje de cooperación = 41\%; Encuesta 2, $\mathrm{n}=574$, enero 1999, porcentaje de cooperación $=35 \%$; Encuesta 3, $n=159$, J unio 1999, porcentaje de cooperación $=54 \%$; y Encuesta 4 , $n=426$, junio 1999, porcentaje de cooperación $=44 \%)$. En cada encuesta, se presentó a los encuestados dos métodos para estimar c y también se les proporcionó algo de información demográfica. El método de escalamiento proporcional necesitó una media de 7 minutos mientras que el método de suma necesitó 5 minutos. De promedio, cada estimación llevó 15 segundos por subpoblación utilizando el método de escalamiento proporcional contra los 18 segundos que se tardó con el método de suma ${ }^{4}$. En todas las ocasiones, las preguntas de escalamiento proporcional precedieron a las cuestiones de suma; esto es una limitación potencial que será remediada en futuras encuestas.

\footnotetext{
${ }^{4}$ Un crítico expresó sus dudas acerca de la baja tasa de cooperación en las 4 encuestas. El peligro de las tasas bajas es que aquellos que no respondieron pueden ser distintos a aquellos que sí. Aunque éste es un motivo válido de preocupación, hay que tener dos cosas en cuenta. Las tasas de respuesta y de cooperación han bajado de forma drástica en las dos últimas décadas. Las tasas detalladas aquí no son inusuales para un estudio nacional que utilice la marcación digital al azar de número telefónicos, sin incentivo ni carta introductoria. En segundo lugar, no parece haber correlación entre el tamaño de la red estimado y el número de intentos requeridos para completar la encuesta. Nuestra conclusión es que más llamadas no habrían cambiado nuestros resultados.
} 
Para las encuestas 1,2 y 4 los números de teléfono se generaron con marcación de dígitos al azar utilizando una base de datos que elimina bancos de teléfonos básicamente comerciales. La marcación de dígitos al azar tiene la ventaja de incluir hogares con números no incluidos en la lista de muestra. Los encuestados dentro del hogar se eligieron preguntando primero por hombre más joven y, si éste fallaba, por la mujer más mayor de la casa. Este método tiende a equilibrar una disposición natural hacia un número desproporcionado de mujeres encuestadas. Las encuestas se realizaron en inglés y en español. Los números de teléfono se descartaron como improductivos a las 10 llamadas y se llamó a los rechazos dos veces.

La encuesta 3 era una muestra de clero comprada a un servicio de muestras conocido a nivel nacional. El clero incluía curas, reverendos y rabinos. Los encuestados fueron seleccionados al azar de la lista utilizando un número asignado al azar por el Sistema de Análisis Estadísticos. Dada la muestra, no se introdujo el elemento azar con la selección del encuestado dentro del hogar. Esta encuesta se realizó en inglés.

\section{Comparación de los valores c}

La Ilustración 1 muestra la distribución de tamaño de la red usando los dos métodos. Los círculos cerrados representan la distribución para el método de escalamiento proporcional en nuestra encuesta original de 796 encuestados (Encuesta 1), mientras que los círculos abiertos representan la distribución por el método de suma (los cuadros abiertos y cerrados se refieren a la encuesta de copia de los 574 encuestados [Encuesta 2], que se detallará más adelante). Una prueba Kolmogorov-Smirnov de igualdad muestra que estas distribuciones son estadísticamente distintas. Visualmente, sin embargo, estas distribuciones parecen remarcablemente similares; de hecho, para la Encuesta 1 , el método de escalamiento proporcional ofrecía un tamaño de la red principal de 290.8 ( $S D=264.4)$ comparado con el 290.7 ( $S D=258.8)$ para el método de suma. La similitud de las medias es tan sorprendente que nos llevó a preguntarnos si podía existir algún efecto instrumental. Si, por ejemplo, los encuestados no pensaron mucho o nada sobre las estimaciones de cuánta gente conocían en cada una de las subpoblaciones o categorías de relación (y simplemente se inventaron resultados similares), esperaríamos que las estimaciones de escalamiento proporcional y de suma fueran similares para casos individuales. La llustración 2 traza las estimaciones de tamaño de las dos redes para la Encuesta 1. Si ambas estimaciones fueran realmente similares, esperaríamos una pequeña dispersión en 
la diagonal. Pero sin embargo, parece existir una variabilidad significativa en todos los niveles del tamaño de la red.

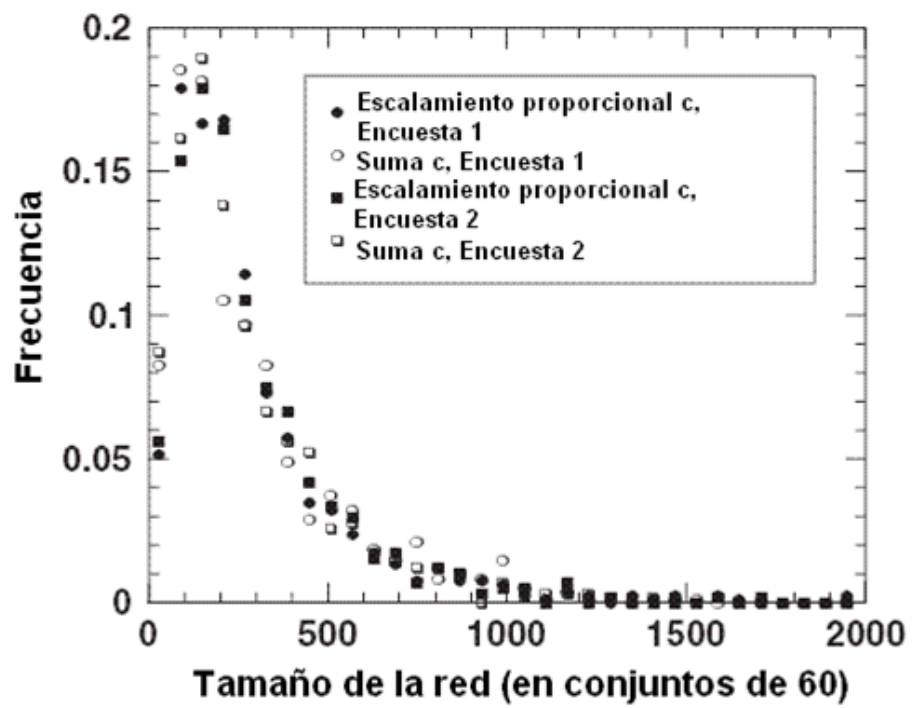

I lustración 1. Frecuencia del tamaño de la red para las encuestas 1 y 2.

Nota: Los símbolos cerrados muestran el tamaño estimado obtenido con el método de escalamiento; los símbolos abiertos, los computados con el método de suma.

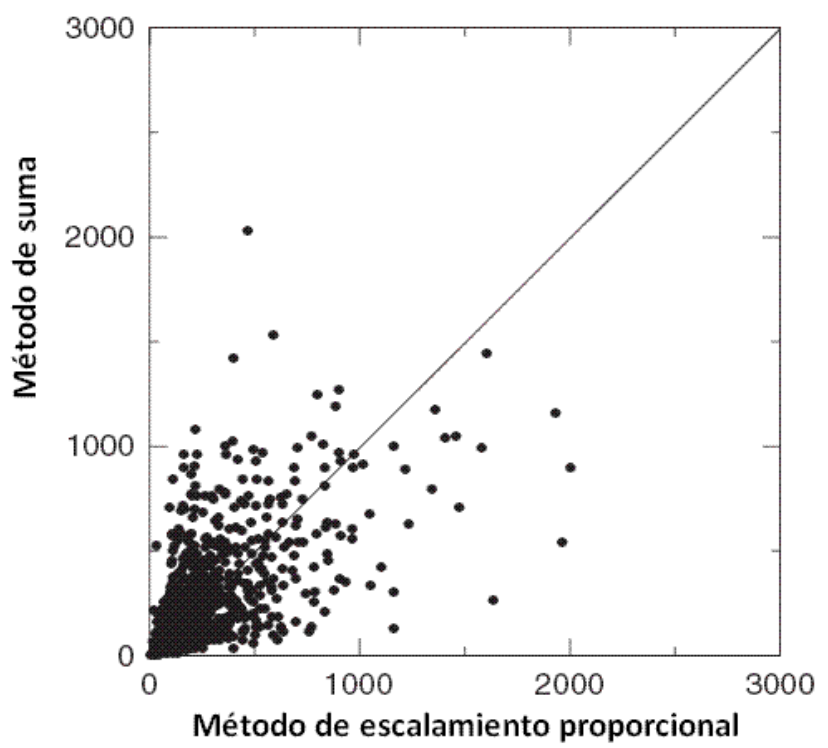

I lustración 2. Estructura de dispersión de las estimaciones de tamaño de la red de la encuesta 1 mostrando las estimaciones del método de escalamiento propocional y del método de suma.

De hecho, la correlación (0.56 a p=0.0001) implica variación entre las dos estimaciones. En otras palabras, a menudo se produce el caso de que una estimación es baja mientras que la otra es elevada. Aún así, en toda la muestra de 796 encuestados para la Encuesta 1 se equilibra para ofrecer resultados similares. Parece ser que los métodos corroboran de forma independiente una estimación de 
291 para el tamaño medio de la red, para la definición de redes de la encuesta. Otra posibilidad es que el encuestado se canse y sea más propenso a dar simples repeticiones a medida que la entrevista progresa ${ }^{5}$. Hay que recordar también, que los encuestados realizaron primero estimaciones sobre cuántas personas conocían en varias subpoblaciones y luego tuvieron que estimar el número de alteri en la red en los varios tipos de relaciones. En el momento de llegar a las últimas estimaciones, es posible que los encuestados hubieran caído en un patrón de repetición. Esto puede no ser la causa de que las estimaciones sean iguales, pero sí es motivo de preocupación.

La Ilustración 3 muestra el coeficiente de variación 4 del número de personas teóricamente conocidas (es decir: m) para cada subpoblación y tipo de relación en el orden en que se realizaron las preguntas. Si los encuestados habían caído en un patrón en el que tendían a dar estimaciones similares para todas las subpoblaciones y tipos de relación a medida que la entrevista progresaba, esperaríamos que la línea fuera más plana. Con la excepción de una línea relativamente plana que se inicia con el octavo nombre de pila y termina con el último nombre de pila, la línea muestra una variación considerable con estas estimaciones. Esto se hace especialmente patente en la categoría de relación "otros", y en las subpoblaciones computables como "gente que recibe diálisis" o "víctimas de homicidio". Parece que los encuestados no caen en el patrón de repetir estimaciones similares a medida que la entrevista progresa. Como prueba, hemos contado el coeficiente medio de variación dentro de cada uno de los tres tipos (nombre, otras subpoblaciones y categorías) para cada encuestado y hemos realizado una media entre todos los encuestados. Los tres promedios (fluctuación estándar) resultaron 1.09, 1.90, 1.55 $(0.45,0.66,0.56)$ que son todos bastante diferentes, cosa significativa.

\footnotetext{
${ }^{5}$ Como el orden de preguntas permaneció constante (es decir, las categorías era lo último que se preguntaba), esto podría haber tenido serias consecuencias.
} 


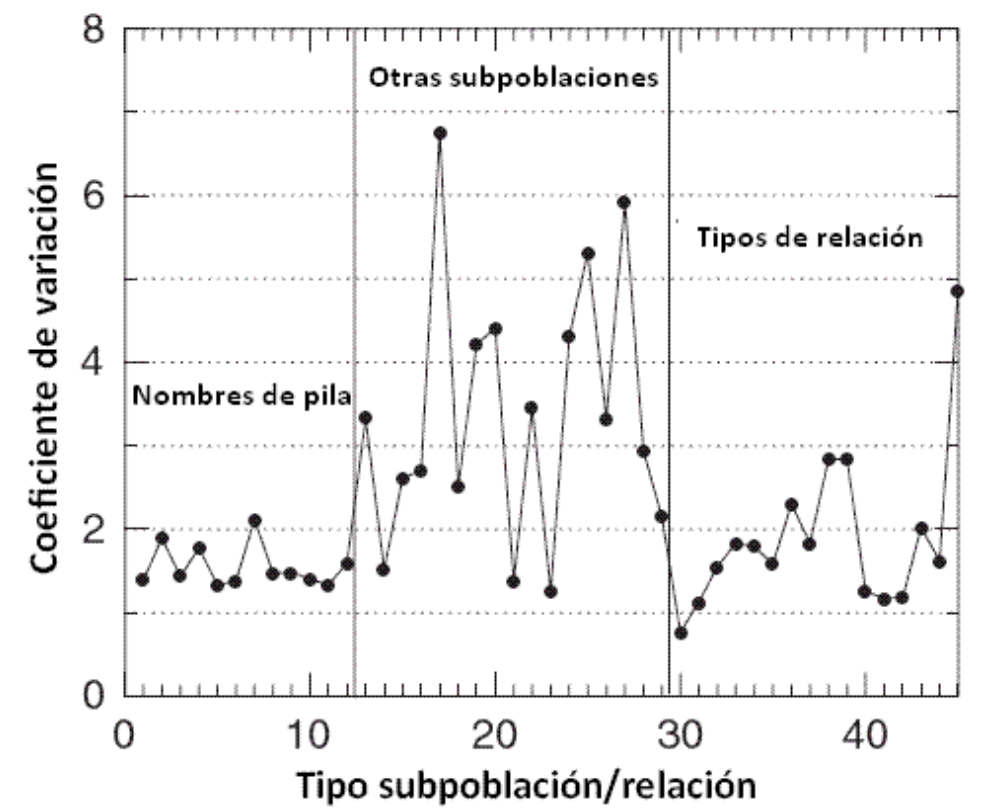

I lustración 3. Coeficiente de variación del promedio de gente teóricamente conocida por subpoblaciones y tipos de relación, por orden de entrevista.

Dada la sorprendente similitud de promedio de tamaño de la red generado por los dos métodos, decidimos reproducir la encuestas con una muestra independiente $(n=574)$, que también se muestra en la llustración 1. En esta muestra, la estimación de tamaño de la red con el método de escalamiento proporcional fue de $291,2(S D=259,3)$ y para el método de suma fue de $281,2(S D=255,4)$. De nuevo, el método de escalamiento proporcional y el método de suma muestran distribuciones esencialmente idénticas, tanto entre ellas como comparadas con la Encuesta 1. Esto parece ser la confirmación sorprendente de la fiabilidad de los métodos. Basándonos en la similitud de las distribuciones de la llustración 1 y en los promedios constantes de tamaño de la red en ambas encuestas, hemos combinado los datos de las dos encuestas $(n=1.370)$ para analizarlos más detenidamente.

La Tabla 1 muestra los resultados. En las 32 subpoblaciones que representan el método de escalamiento proporcional de la Tabla -incluyendo tres subpoblaciones para las que no es posible la corroboración independiente del tamaño- la $r$ de Pearson para los informes promedio de ambas encuestas es de 0.99. De forma similar, utilizando los números proporcionados en las 16 subpoblaciones que representan el método de suma en la Tabla 1, la $r$ de Pearson para ambas encuestas es de 0.99 (hay que apuntar, por supuesto, que estamos correlacionando elementos que tienden a covariar, por lo que cabe esperar tanta correlación). 
Las estimaciones también se pueden realizar para tamaños de subpoblación utilizando los métodos de Killworth et al. (1998b), que mostraron que las estimaciones aumentan de forma proporcional al número medio obtenido en el promedio de tamaño de la red personal. Ambas cifras son prácticamente idénticas en ambas encuestas y a consecuencia de ello, las estimaciones secundarias de tamaño de la red también son idénticas (y por lo tanto no se muestran).

La consistencia entre ambos métodos -en la distribución visual del tamaño de la red y las estimaciones de tamaños de subpoblación- sugirieron que continuáramos examinando el proceso por el cual la gente realiza estas estimaciones ante la pregunta que se le formula. Por falta de un instrumento estándar de medición del tamaño de la red y de su distribución, no podemos determinar cuál de los dos métodos produce unos resultados más fiables y válidos. Por ello decidimos que necesitábamos más información sobre los procesos reales por los que pasan los encuestados cuando proporcionan sus estimaciones. Una forma obvia de adquirir esta información es trabajar con grupos focales. El grupo focal es una herramienta valiosa para extraer información sobre el proceso porque se aprovecha de las interacciones dentro del grupo - mientras un encuestado discute un tema, otros encuestados escuchan lo que dice; esto impulsa un debate y las réplicas de otros encuestados.

\section{Grupos focales}

Trabajamos con dos grupos focales en Gainesville, Florida: un grupo de 9 hombres y un grupo de 7 mujeres. Las edades de ambos grupos oscilaban entre los veinte y más de sesenta años. Los encuestados de los grupos focales fueron reclutados por un estudiante en aparcamientos y centros comerciales. Se preguntó a los encuestados si estaban dispuestos a venir a la Universidad, responder a una encuesta y someterse a una entrevista de unas dos horas. Los que aceptaron participar cobraron 75 US\$. Los participantes respondieron primero a las preguntas de forma presencial ante uno de los entrevistadores que había realizado la encuesta telefónica. Queríamos que los participantes experimentaran el proceso de la misma forma que los encuestados telefónicos. Durante las sesiones con los grupos focales nos centramos en las siguientes cuestiones: ¿Cuáles han sido sus impresiones generales sobre la encuesta?; ¿Le ha parecido extraña?; ¿Se ha sentido incómodo respondiendo a alguna de las preguntas?; "Describa el proceso por el que ha pasado al estimar el número de personas que conoce llamadas Michael"; ¿Cree que su estimación ha sido exacta?; ¿Cree que ha olvidado a alguien?; ¿Ha tenido tiempo suficiente para proporcionar sus estimaciones?; ¿Han sido algunos grupos 
más difíciles de estimar que otros?; ¿Le parece razonable la definición de "conocer a alguien"?, ¿Cree que la definición deja fuera a personas importantes?; ¿Los tipos de relación utilizados se ajustan a su red personal? ¿Cree que algunas categorías son demasiado amplias para estimarlas?

En general, los participantes de los grupos focales se interesaron por el estudio. Se eligieron las 29 subpoblaciones computables de la Tabla 1 porque representan a una variedad de tipos de subpoblación sobre las que se recogen estimaciones fiables cada año. Algunos de los hombres, sin embargo, no se fiaban de nuestras intenciones cuando les preguntamos a cuántos vendedores de armas conocían.

La mayoría de los participantes sintieron que nuestra definición de "conocer" era adecuada, aunque les preocupaba que algunos de sus alteri importantes en la red quedaran fuera por el límite de 2 años. Aunque no habían hablado con algunas personas en dos años, algunos participantes dijeron que podrían restablecer la relación en el punto en que la habían dejado. Pero la mayoría de los participantes estuvieron de acuerdo en que estos contactos no afectaban mucho a sus vidas.

Las revelaciones más valiosas sobre el proceso de respuesta de las preguntas de nuestra encuesta llegaron cuando perdimos a los participantes en los grupos focales que recordaran el proceso por el que habían pasado al estimar los tamaños de subpoblaciones específicas en sus redes personales.

Todos los participantes estuvieron de acuerdo en que para algunas subpoblaciones, básicamente las más grandes (por ejemplo, gente llamada Michael), era difícil realizar la estimación en tan poco tiempo.

Varias personas mencionaron la diferencia entre contar y estimar (un tema tratado por Sudman et al. 1996). Para grupos relativamente pequeños, como los vendedores de armas, dijeron que enumeraban; para otros, como nativos americanos o gente llamada Michael, calculaban una estimación. Algunos participantes dijeron de forma explícita que confiaban en su "intuición" para calcular el tamaño del grupo y a cuánta gente conocían en el grupo. Cuando se les preguntó qué números utilizaban para hacer efectiva esa intuición, se hizo evidente que los números variaban ampliamente entre los participantes. 
El hallazgo con los grupos focales de que los encuestados conjeturaban sobre algunas subpoblaciones, y de que algunos encuestados no dijeron que utilizaban el mismo valor de forma repetida, nos llevó a examinar los datos de la encuesta para encontrar pruebas de preferencias numéricas. Si los encuestados seleccionaban la conjetura sistemática en lugar de calcular en muchas de las subpoblaciones, y si estos valores de conjetura variaban en gran medida entre encuestados, esto podía explicar las estimaciones similares para tamaños de la red entre las respuestas del método de escalamiento proporcional y entre el método de escalamiento proporcional y el método de suma. Aunque parecen fundamentalmente distintos, ambos métodos dependen de la estimación de los encuestados respecto al número de gente que conocen en una categoría determinada. La estimación sistemática no relacionada con el tamaño de la red sería un error grave. Por otro lado, la conjetura podría ser sistemática, pero relacionada con el tamaño de la red.

\section{Preferencia numérica}

Redondear a valores prototípicos es el fenómeno por el cual la gente, cuando se les pide aportar un número como una cuenta o una estimación en una categoría, responde con números enteros preferidos (Myers 1940; Turner 1958; Zelnick 1961, 1964; Winick 1962; Stockwell 1966; Stockwell y Wicks 1974; Wicks y Stockwell 1975; Huttenlocher et al. 1990; Baker 1992). Algunos valores proporcionados de forma habitual pueden ser $0,5,10,15,20,25,30,35,40,45,50 \ldots 100 \ldots 200$, lo que muestra una preferencia por los dígitos terminales $0,5,00$. Sin embargo, normalmente, la serie de valores preferidos puede incluir otros números, como el 2 , el 8 y el 12. Para abreviar adoptaremos un término simple ya usado anteriormente (Myers 1940; Turner 1958) y nos referiremos al fenómeno del redondeo como heaping, y a los valores que muestran este fenómeno como a valores heap. La tendencia a repetir números preferidos ocurre normalmente en conjunción con la marginación de ciertos otros números, como el 7 , el 9, el 11, el 13, el 14 y el 16. En una estimación simple, este último fenómeno muestra que típicamente la gente suele evitar los dígitos terminales 1 y 9 , a veces el 3 y el 7 , y posiblemente el 4 y el 6 (Turner 1958).

La Ilustración 4 muestra un ejemplo de heaping. La llustración muestra el número total de aportaciones de $0,1,2,3,4 \ldots$ de todos los subgrupos de la primera encuesta, utilizando una escala logarítmica, que incluye el cero por razones de claridad (ya que la respuesta más común es 0 ). Los valores selectos muy altos en comparación con aquellos a su alrededor están indicados en el diagrama. Queda claro que los encuestados prefieren los números con dígitos terminales 0 y 5 , 
especialmente para las respuestas mayores de 10; y que prefieren dejar sin utilizar otros números (como 7, 9, 11). Entre los números mayores de 20 predominan los múltiples de 5. Aunque Roberts and Brewer (2000) presentan algunas medidas de heaping en datos de red, en nuestros datos se ve claramente que se produce heaping. ¿Podría ser que el heaping modifique nuestros resultados? A la vista de ello, parece difícil rectificar los efectos del heaping, ya que sólo observamos sus resultados y no las condiciones antes de que los números se hayan modificado por la preferencia. Y los grupos focales nos han aportados pocos datos sobre cómo se llevó a cabo el proceso de heaping. Hemos tratado de analizar los efectos del heaping de dos formas: 1) creando un modelo de cómo pueden cambiar los datos, y de cómo nuestros resultados cambiarían, utilizando datos iniciales plausibles para los datos de suma; y 2) con una variedad de métodos ad hoc para invertir el heaping dados los datos de escalamiento proporcional finales.

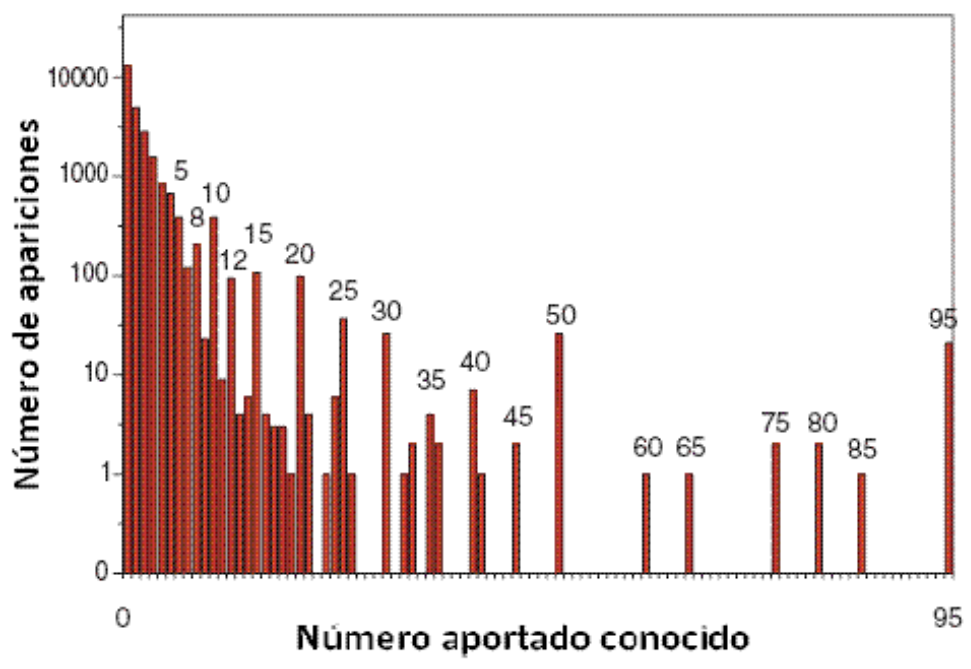

I lustración 4. Número de apariciones de número aportado conocido de cualquier subpoblación o tipo de relación por 796 encuestados.

Nota: el eje y está aumentado de forma logarítmica pero incluye cero. Esto incha algunos de los valores pequeños a nivel visual, pero no se puede evitar dado el ampio rango de los valores

\section{Modelando cómo podrían cambiar los datos y cómo cambiarían nuestros resultados.}

Existen pruebas claras (detalladas más abajo) de que el heaping en la Ilustración 4 tienen poco efecto sobre las estimaciones aportadas por el método de escalamiento proporcional. Sin embargo, esto no tiene por qué ser cierto para las categorías relacionales, ya que se suman para proporcionar una estimación c, cosa que puede agravar los errores. Para investigar posibles efectos, hemos elaborado una 
distribución de frecuencia ad hoc $\mathrm{f}(\mathrm{k})$ del número de respuestas para cada valor de k, $0<\mathrm{k}<134$, como se muestra en la Tabla 2.

\begin{tabular}{ll}
$\mathbf{K}$ & $\mathbf{f ( \mathbf { k } )}$ \\
\hline 0 & 268 \\
1 & 135 \\
2 & 69 \\
3 & 37 \\
4 & 21 \\
5 & 13 \\
6 & 10 \\
7 & 9 \\
8 & 8 \\
9 & 6 \\
$10-12$ & 5 cada uno \\
$13-20$ & 4 cada uno \\
$21-35$ & 3 cada uno \\
$36-68$ & 2 cada uno \\
$69-134$ & 1 cada uno
\end{tabular}

Tabla 2. Distribución de frecuencia para una distribución ad hoc de respuestas para la típica categoría relacional.

El número total de encuestados es de 800, que es parecido al de la Encuesta 1. Esta función de frecuencia es monótonamente decreciente, comenzando como un exponencial negativo pero que poco a poco se va allanando hasta que no hay cola no trivial fuera del valor más alto. Es una distribución idealizada, similar a aquellas halladas en las respuestas relativas a las categorías relacionales en la Encuesta 1. Para las últimas subpoblaciones, los primeros valores eran similares, las distribuciones decrecían de forma parecida, y se veían colas no triviales saliendo por lo menos hasta como muestra la Ilustración 4, pero con valores altos ocasionales debido al heaping. La respuesta media para esta distribución idealizada es 16.09 , mientras que la respuesta media para las 16 categorías relacionales es 19.54.

En nuestras encuestas los valores máximos impuestos para codificar las respuestas para las distintas subpoblaciones se establecieron en 95 para las 12 subpoblaciones de nombre y las 20 de atributo, y variaban entre 99, 100, 150 y 500 para las 16 categorías relacionales. Parece que algunas respuestas reales podrían haber excedido esos valores máximos, porque las altas frecuencias para algunos de ellos parecen desproporcionadas respecto a las respuestas para los valores heap menores y por lo tanto, podrían ser una invención. Este efecto también se integrará en el modelo utilizando esta distribución ad hoc. 
Ahora, supongamos que esta distribución idealizada es la verdadera distribución subyacente para los encuestados, pero que los encuestados dan valores heap por defecto. ¿Cómo se produce el heaping? El siguiente mecanismo es un posible modelo cognitivo para este fenómeno. Supongamos que tenemos los dos valores heap adyacentes 10 y 25 , donde los encuestados no proporcionan ningún número entre estos dos si no que más bien se inclinan hacia los valores heap por defecto más próximos. Si un encuestado tiene un número "real" de gente que conoce en una subpoblación (según la distribución idealizada) que se encuentra en la primera mitad del intervalo 11-24, principalmente 11-17, el encuestado proporciona un 10, y si se encuentra en la segunda mitad, entre 18-24, da como respuesta un 25 . Si el intervalo tiene unos valores impares, asumimos que los valores medios se ajustan por defecto al valor heap inferior la mitad de las veces y al valor superior la otra mitad. Asumimos entonces que los valores por defecto de más arriba ocurren entre cada par de valores heap adyacentes, y que los valores "reales" que ya son valores heap no cambian sino que los encuestados los proporcionan de forma exacta.

Comparamos la respuesta media para la distribución por defecto que contiene valores heap con la distribución idealizada de la que se deriva. Para los valores fijos t y e el porcentaje de cambio de la media m se aproximará al porcentaje de cambio del valor estimado de c de (1). Asumimos que los valores heap aquí son aquellos menores o iguales a 100, que aparecen al menos en la mitad de las 16 categorías relacionales en la Encuesta 1, y estimamos el efecto del heaping en la media $\mathrm{m}$.

Para los valores heap $0,2,5,8,10,12,15,20,25,30,40,50$ y 100, nos encontramos que la media $\mathrm{m}$ es $15.08,93.8 \%$ del original 16.07 , para un error de alrededor del $6.2 \%$. El valor estimado resultante de c sería por lo tanto menor, por poco más del $6 \%$.

Otras combinaciones de valores heap de la lista anterior para las categorías relacionales muestran errores similares. Este ejemplo también incorpora los efectos de invención que aparecieron en algunos casos dentro de los datos relacionales; básicamente, imposición de valores máximos (heap) que eran demasiado bajos para codificar las respuestas reales. En este caso, todas las respuestas por encima del valor máximo se redondean a la baja, produciendo un valor bajo ficticio para c para aquellas categorías relacionales en las que esto ocurrió. Se están estudiando otros escenarios para el heaping, que se detallarán más adelante (Johnsen et al. n.d.). 


\section{Métodos ad hoc para invertir el heaping en el método de escalamiento proporcional}

Tratamos de deshacer cualquier efecto de conjetura (sea redondeado o no) cambiando cada respuesta dada por un número al azar distribuido de forma uniforme en el campo 50-150 por ciento de las respuestas dadas. En otras palabras, asumimos que los encuestados conjeturan de forma imparcial. Casi no se detectaron cambios en la media c (291.0), y no se cambio ninguna estimación de ningún tamaño de subpoblación más de un $5 \%$, normalmente menos.

Como este cambio era mayor que el conseguido simplemente modificando las respuestas redondeadas, llegamos a la conclusión de que el heaping tiene un efecto muy limitado sobre nuestros resultados, y que, por lo tanto, no les afecta esta forma de error.

Sin embargo, el momento y el lugar en el que se produce el heaping pueden tener un efecto. Considerando (3) en Killworth et al. (1998b: 293) la estimación de probabilidad máxima para la c de cada encuestado:

$$
\tau_{t}=t \cdot \frac{\sum_{f=1}^{L} m_{i f}}{\sum_{f=1}^{L}}
$$

donde el sufijo $\mathrm{i}$ se refiere al encuestado y $\mathrm{j}$ a cada una de las subpoblaciones $\mathrm{L}$ (por lo que $\mathrm{m}_{\mathrm{ij}}$ es el número de personas encuestadas que dicen conocer a $\mathrm{j}$ personas en la subpoblación j, con un tamaño dentro de la población total de ej). Podríamos asumir que las respuestas de los encuestados no son fiables para los valores por encima del valor límite k. Para expresarlo de forma simple ${ }^{6}$, siempre que la $m_{i j}$ esté por encima de $k$, multiplicamos ambos resultados de la suma de $m_{i j}$ $y$ el respectivo $e_{j}$ en el numerador y denominador por un valor arbitrario de 0.2 (más que una unidad implícita). Hallamos que los resultados son muy dependientes de este cambio en el método. Incluso con un límite de 10, la media c cae a 233, un $20 \%$ menos. En otras palabras, para reproducir nuestros hallazgos, las respuestas grandes (que corresponden a los encuestados con grandes c y con grandes subpoblaciones) deben contar con todo su peso. La dependencia de las soluciones

\footnotetext{
6 Una aproximación estadística más exacta incluiría ratios de variabilidad de error, que aquí se desconocen.
} 
en las subpoblaciones elegidas para el estudio se discutirá más ampliamente en otro artículo.

\section{La coherencia entre estimaciones c no es ficticia}

Hemos hablado de hallazgos que muestran una gran coherencia entre dos métodos distintos para contar $c, y$, por lo tanto, también en estimaciones secundarias de tamaños de subpoblación. Sin embargo, ambos métodos comparten la necesidad de pedir a los encuestados que estimen el número de personas conocidas en varias subpoblaciones: en un caso, subpoblaciones seleccionadas (de escalamiento proporcional) y en el otro, categorías de relación (de suma). Nos preocupaba, por lo tanto, que la concordancia fuera ficticia: esencialmente, casi cualquier respuesta obtenida podría haber dado resultados similares tras procesar los datos. Para probar esto, examinamos los efectos de los cambios en los datos de los resultados, básicamente el promedio de c.

Hemos argumentado que los encuestados podrían conjeturar sus respuestas cuando su número estimado sobrepasa un valor límite. Ahora, calculamos que si los encuestados estimaron números a partir de 5 , el número medio estimado era de 5,24 . Por lo tanto cambiamos los valores $m$ proporcionados de 5 o más a un valor de 5 exacto. Esto produjo grandes cambios, e hizo descender la media c a 206, un cambio del $29 \%$. Los cambios a las estimaciones de tamaño de subpoblación eran similares, variando en tamaño y en dirección (las estimaciones para las subpoblaciones pequeñas crecieron, ya que el conocimiento sobre éstas no cambió en los datos y sólo la estimación media de c había cambiado).

Esto se repitió, sustituyendo los valores de más de 5 por 10 (es decir, doblando prácticamente el valor medio proporcionado sobre 5). El promedio c no sufrió apenas cambios, a 284. Una respuesta similar se halló al sustituir los valores de más de 5 por un valor distribuido al azar de forma uniforme entre 5 y 15. Repetimos el cambio al azar (5-15) detallado anteriormente, pero sólo en las subpoblaciones grandes (con e > 1 millón). Esto hizo aumentar el promedio c a 402, un cambio del 38\% pero en la dirección opuesta. De nuevo, las estimaciones de subpoblación cambiaron con una proporción similar. Entonces realizamos otro cambio, más pequeño pero sistemático. Sustituimos las estimaciones de subpoblación por encima de 10 por 10. La media c cayó a 245 , un cambio del $16 \%$.

Estos experimentos muestran que algunos cambios en los datos pueden producir grandes alteraciones en los resultados. Cambios aparentemente pequeños (sustituir m por 5 cuando es superior a 5, cuando la media existente de estos datos nos da 
5.24) produce un gran cambio en el resultado. Al contrario, algunos cambios aparentemente importantes en los datos (cambiar m por 10 en este caso) produce un cambio menor, al menos en el promedio c. Por lo tanto, podemos rechazar fehacientemente la sugerencia de que "cualquier estimación razonable de subpoblación grande produciría resultados similares con este método".

\section{Datos que faltan}

En toda encuesta se da el caso de que a menudo los encuestados no saben, o no contestan, a algunas preguntas. Por ejemplo, dependiendo del entorno y las características de un determinado encuestado, quizás no sea capaz de estimar el número de gente que conoce en una población (como gente en su organización o gente llamada Michael) mientras que sí son capaces de estimar otras (como la gente en su familia inmediata o gente llamada Nicole). Hemos estimado de distintas formas qué efecto podría tener esto en nuestros resultados. Primero, comparamos estimaciones de c y estimaciones secundarias de tamaños de subpoblaciones utilizando el método de escalamiento proporcional, que trataban los datos omitidos de dos formas.

El primer método (utilizado con todos los datos proporcionados en nuestros artículos anteriores), cuando añadía un valor $m$ y un valor e a alguna subpoblación en el denominador, ignoraba ambas contribuciones en (2) arriba, si no había datos para esta subpoblación (es decir, si el encuestado había ignorado esta subpoblación $)^{7}$.

El segundo método seguía añadiendo el valor e en el denominador, pero no cambiaba el numerador. El cambio resultante era del $1 / 3 \%$ para la c de todos los encuestados (aunque alcanza un máximo del $30 \%$ en muy pocos encuestados). Por lo tanto, los datos que faltan tienen un efecto insignificante sobre el método de escalamiento proporcional.

No se da el mismo caso para el método de suma. Faltaban datos en una o más categorías de relación utilizadas para calcular la suma c en un 35\% de los 1.370 encuestados para las encuestas 1 y 2 (25\% de los 1.370 no habían respondido una o dos categorías, el restante $10 \%$ no había respondido en más de dos). Los resultados de la suma de c citados arriba simplemente sumaban los valores aportados (ignorando los datos que faltaban). Reemplazando estos datos con

\footnotetext{
${ }^{7}$ Las matemáticas que llevan a la formula de probabilidad máxima (2) muestran que éste es el enfoque correcto.
} 
medias en todas las respuestas de los encuestados para esa categoría, hallamos una falta de información de 24 , o $8 \%$ de la media c. Por lo tanto, los datos omitidos y los efectos del heaping se pueden combinar para producir una omisión de información en el método de suma, y es necesario asegurarse de que los encuestados respondan todas las preguntas cuando se utiliza este método.

\section{Encuestados con redes atípicamente grandes}

Otra forma de probar la validez de nuestro método para estimar tamaños de las redes personales es restringir a nuestros encuestados a subpoblaciones cuyo tamaño de red esperamos sea muy grande o muy pequeño. Como ya hemos señalado, la literatura sobre estimación de tamaño de la red personal es limitada, y no existe virtualmente nada sobre tamaños de redes en subpoblaciones particulares.

Por lo tanto, debemos confiar en la experiencia y en el sentido común para sugerir subpoblaciones apropiadas para esta prueba.

Otro requisito para la subpoblación es que debe existir una muestra disponible. Aunque nos gustaría, no podemos comprar una muestra de reclusos o ermitaños. Las subpoblaciones que creemos tienen mayores redes incluyen a los políticos, los representantes sindicales, los licenciados y el clero. Compramos una lista de teléfonos de una muestra representativa de miembros del clero de todo el país y la utilizamos para llevar a cabo la encuesta 3 descrita anteriormente. Para estos datos, el tamaño medio de las redes para el método de escalamiento proporcional era de $(S D=504)$ y para el método de suma, $948(S D=1.223)$. La diferencia entre el tamaño de redes para el clero comparado con el de la población general es significativo $(p=0.0001)$. Como esperábamos, el tamaño medio de la red producido por ambos métodos era más grande, con diferencia, que aquellos para la población general.

Parece que hay una gran diferencia entre el promedio del tamaño de la red del clero generado por los dos métodos. La prueba A revela que estas dos estimaciones son significativamente distintas $(p=0.0001)$, mientras que eran estadísticamente las mismas en ambas encuestas 1 y 2 . Lo más probable es que esto esté causado por los valores más grandes de una o dos categorías de relación utilizadas para computar la suma del tamaño de red, específicamente aquellas en las que se pide a los encuestados a cuánta gente conocen a través de organizaciones y afiliaciones religiosas. Hay que apuntar, sin embargo, que la estimación para los tamaños de red del método de escalamiento profesional es también mayor para el clero que 
para la población general, y que este método no contiene subpoblaciones que tiendan de forma obvia a generar grandes estimaciones por parte del clero. Hay que notar también que el hecho de que algunas clases de encuestado puedan generar mayores tamaños de red indica que nuestros hallazgos coherentes en muestras representativas a nivel nacional no son un invento de nuestra metodología, al menos en los EEUU.

\section{Rango de Datos}

Hasta ahora no tenemos conocimiento de las habilidades de los encuestados para proporcionar de forma exacta la información que requerimos. Los grupos focales y nuestro estudio del heaping indicaron que los encuestados resolvían el problema de proporcionarnos una estimación de dos formas, dependiendo de la cifra que estaban estimando: cuando era pequeña, enumeraban; cuando era grande, estimaban. Como creíamos que podría ser útil preguntar al menos a los encuestados si creían que sus respuestas eran exactas, llevamos a cabo la encuesta 4.

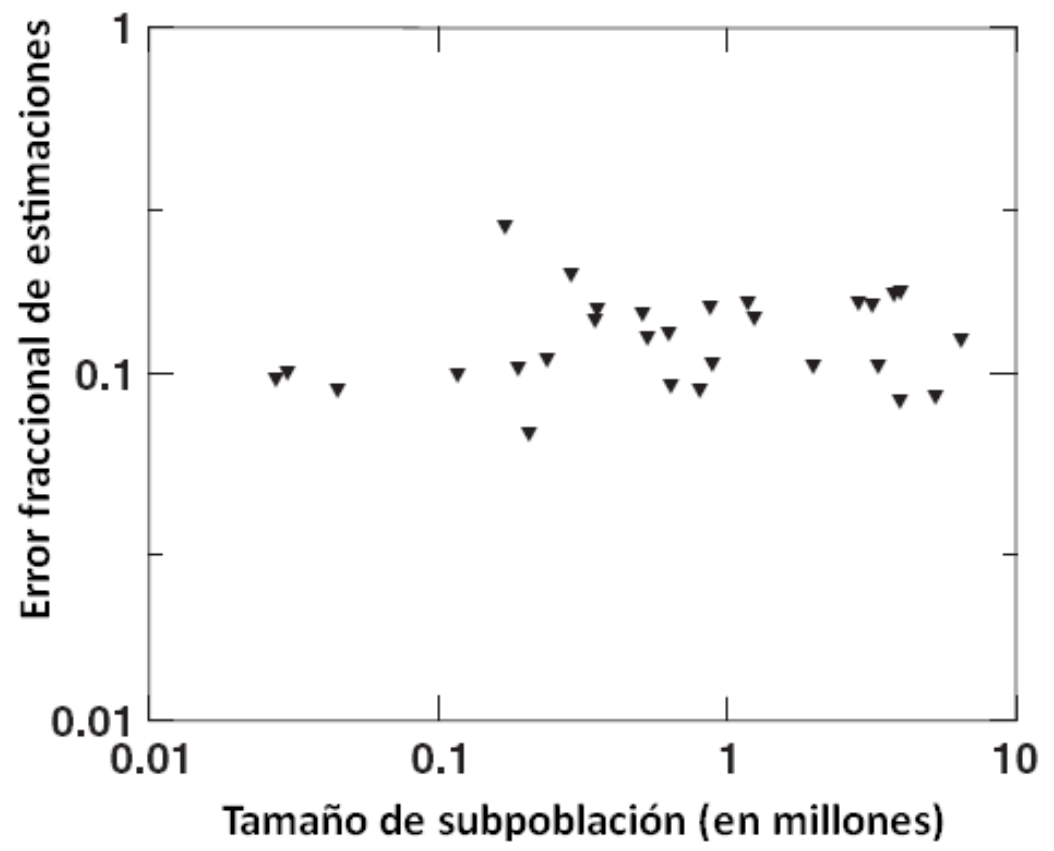

I lustración 5. Media de error fraccinal de número estimado conocido en subpoblaciones de la encuesta 1, como función de tamaño de subpoblación conocida.

Nota: No existe ninguna correlación significativa.

El cambio producido fue que en lugar de preguntar a cuánta gente conocían en una subpoblación, pedimos a los encuestados que nos proporcionaran un rango, consistente en un número mínimo y un máximo, entre los cuales estaban seguros que encajaba su respuesta. Sospechamos que las respuestas de los encuestados 
confirmarían nuestras sospechas: para las subpoblaciones pequeñas obtendríamos una respuesta bastante precisa -es decir, alcances fraccionarios bajos- mientras que para las subpoblaciones grandes obtendríamos una respuesta imprecisa con un alcance fraccionario alto. Para nuestra sorpresa, no fue así. La llustración 5 muestra el alcance fraccionario de los números proporcionados conocidos en cada subpoblación para la primera encuesta como una función del tamaño real de la subpoblación. No demuestra una variación significativa en el alcance fraccionario. Por ello, los encuestados o pensaron que eran más o menos exactos (como fracción del tamaño de subpoblación) con las subpoblaciones más o menos grandes, y descartamos esto como una posible causa de error.

\section{Discusión}

Hemos descrito dos métodos para estimar el tamaño de las redes personales y hemos mostrado que ambos generan distribuciones similares. Los resultados se repiten a lo largo de varias réplicas independientes. Además, no pudimos encontrar ningún efecto instrumental que explicara estos resultados similares y coherentes. Por ello debemos concluir que ambos métodos generan estimaciones que, a lo peor, son representativas de los tamaños de las redes personales tal como las hemos definido.

Entonces, ¿cómo deberían aplicarse estos métodos? Primero, se pueden usar para estimar el tamaño de la red personal en sí sola. Desde el momento en que el tamaño de la red afecta a (o está relacionado con) los atributos individuales y comportamientos, estos métodos tienen aplicaciones inmediatas.

El tamaño de la red representa acceso a información y recursos. Por ejemplo, aquellos con redes personales extensas pueden estar mejor informados sobre sus opciones de seguro médico. Aquellos cuyas redes están conectadas vagamente y consisten básicamente en amistades y compañeros de trabajo quizás obtengan asistencia pública más rápidamente que aquellos cuyas redes están conectadas de forma densa con grupos de parientes y amigos íntimos (véase: La teoría de la “fuerza de los lazos débiles" de Granvotter 1973).

La segunda aplicación es la estimación de poblaciones difíciles de contar (ver Killworth et al. 1998b para un debate más completo sobre este método). Podemos imaginar muchos usos para un método así. Existen muchas poblaciones ocultas en los países desarrollados que son elusivas, como los heroinómanos o los sin techo. En los países en vías de desarrollo, muchas poblaciones son difíciles de contar porque la infraestructura imposibilita llevar a cabo lo que en EEUU y Europa sería 
una encuesta sencilla. Un método que permita a los investigadores estimar el tamaño de la población difícil de contar de forma rápida y barata y con exactitud razonable no tendría precio.

Nuestro método para estimar el tamaño de la población difícil de contar requiere no sólo una representación para el tamaño de red, si no una estimación exacta del tamaño de red. Aunque estamos seguros de que nuestras estimaciones son representaciones válidas ${ }^{8}$, basadas en los análisis presentados aquí, los hallazgos recientes han puesto en duda la exactitud de estas estimaciones. Otras partes de nuestro análisis (no detalladas aquí) han suscitado dudas sobre el proceso cognitivo por el que pasa un encuestado mientras está calculando estimaciones del número de gente que conoce en varias subpoblaciones y categorías de relación. De forma específica, el promedio conocido en una subpoblación determinada cambia aproximadamente a proporción de la raíz cuadrada del tamaño de la subpoblación. Este patrón contraviene la presunción (1) que predeciría una variación lineal, y en estos momentos estamos estudiando esto. Hasta que entendamos los efectos de esta relación en forma de ley potencial, hay que ser cautos en la aplicación de los métodos para estimar el tamaño de la red en las poblaciones difíciles de contar.

Nuestra conclusión es que ambos métodos para estimar el tamaño de la red son representativos válidos y fiables para tamaños de red reales, pero quedan pendientes las cuestiones sobre la exactitud.

\section{Referencias bibliográficas}

Baker, Michael (1992) “Digit Preference in CPS Unemployment Data". Economics Letters 39: 117-121.

Bernard, H. Russell, Eugene C. Johnsen, Peter D. Killworth, y Scott Robinson (1989) "Estimating the Size of an Average Personal Network and of an Event Subpopulation. In The Small World." Manfred Kochen,ed. pp. 159-175. Norwood N.J .: Ablex Publishing Corporation.

(1991) "Estimating the Size of an Average Personal Network and of an Event Subpopulation: Some Empirical Results". Social Science Research 20:109-121.

Bernard, H. Russell, Peter D. Killworth, y Chris McCarty (1982) "INDEX: An Informant-Defined Experiment in Social Structures". Social Forces 61:99-133.

Brewer, Devon D. (1993) "Patterns in the Recall of Persons in a Student Community". Social Networks 15:335-359.

(1995) "The Social Structural Basis of the Organization of Persons in Memory". Human Nature 6:379-403.

8 Los encuestados creían que el tamaño de la red personal era otra representación válida, ya que generalmente es monótonamente creciente con la probabilidad de conocer a alguien en una subpoblación determinada (Bernard et al. 1989). 
(1995) "Patterns in the Recall of Persons in a Department of a Formal Organization". J ournal of Quantitative Anthropology 5:255-284.

Brewer, Devon D., y Bihchii L. Yang (1994) "Patterns in the Recall of Persons in a Religious Community". Social Networks 16:347-379.

Fowler, Floyd Jackson, Jr. (1992) "How Unclear Terms Affect Survey Data". Public Opinion Quarterly 56:218.

Freeman, Linton C., y Claire R. Thompson (1989) Estimating Acquaintanceship Volume. In The Small World. Manfred Kochen, ed. Pp. 147-158. Norwood N.J.: Ablex Publishing Corporation

Granovetter, Mark S. (1973) "The Strength of Weak Ties". American Journal of Sociology 78: 1360-1380.

Huttenlocher, Janellen, Larry V. Hedges, y Norman M. Bradburn (1990) "Reports of Elapsed Time: Bounding and Rounding Processes in Estimation". Journal of Experimental Psychology: Learning, Memory and Cognition 16: 196-213.

Johnsen, Eugene C., H. Russell Bernard, Peter D. Killworth, Gene A. Shelley y Chris McCarty (1995) "A Social Network Approach to Corroborating the Number of AIDS/HIV+ Victims in the U.S". Social Networks 17:167-187.

Killworth, Peter D., y H. Russell Bernard (1978) "The Reverse Small-World Experiment". Social Networks 1:159-192.

Killworth, Peter D., Eugene C. Johnsen, H. Russell Bernard, Gene A. Shelley, y Chris McCarty (1990) "Estimating the Size of Personal Networks". Social Networks 12: 289-312.

Killworth, Peter D., Eugene C. Johnsen, Chris McCarty, Gene A. Shelley, y H. Russell Bernard (1998a) "A Social Network Approach to Estimating Seroprevalence in the United States". Social Networks 20:23-50.

(1998b) "Estimation of Seroprevalence, Rape and Homelessness in the U.S. Using a Social Network Approach". Evaluation Review 22:289-308.

Myers, Robert J. (1940) "Errors and Bias in the Reporting of Ages in Census Data". Transactions of the Actuarial Society of America 41: 395-415.

Roberts, John. M., and Devon D. Brewer n.d. Measures and Tests of Heaping in Discrete Quantitative Distributions. J ournal of Applied Statistics. En imprenta.

Stockwell, Edward G. (1966)" Patterns of Digit Preference and Avoidance in the Age Statistics of Some Recent National Censuses: A Test of the Turner Hypothesis". Eugenics Quarterly 13:205-208.

Stockwell, Edward. G., y Jerry. W. Wicks (1974) “Age Heaping in Recent National Censuses". Social Biology 21:163-167.

Sudman, Seymour, Norman M. Bradburn y Norbert Schwarz (1996). Thinking About Answers: The Application of Cognitive Processes to Survey Methodology. San Francisco: Jossey-Bass.

Turner, Stanley H. (1958) "Patterns of Heaping in the Reporting of Numerical Data". Proceedings of the Social Statistics Section, American Statistical Association, Washington D.C.:248-251.

Wicks, Jerry W., y Edward G. Stockwell (1975) “Age Heaping in Recent National Censuses: An Addendum". Social Biology 22:279-281.

Winick, Charles (1962) "Preference for Individual Digits". Journal of General Psychology 67:271-281. 
Zelnick, Melvin (1961) "Age Heaping in the United States Census: 1880-1950". Milbank Memorial Fund Quarterly 39:540-573.

(1964) "Errors in the 1960 Census Enumeration of Native Whites". Journal of the American Statistical Association 59:437-459. 